\title{
THE FREIHEITSSATZ FOR ONE-RELATION MONOIDS ${ }^{1}$
}

\author{
C. SQUIER AND C. WRATHALL
}

Abstract. We give an elementary proof of the Freiheitssatz for one-relation monoids.

The Freiheitssatz is basic to the study of one-relator groups. It states that for a group $G$ presented by generators $X$ and single (cyclically reduced) defining relator $r$, if $Y \subseteq X$ excludes some generator occurring in $r$ then the subgroup of $G$ generated by $Y$ is freely generated by $Y$. The analogous property is true of one-relation monoids, that is, of Thue systems with a single rule. A proof of this fact by appeal to the Freiheitssatz for groups $[\mathbf{4}, \mathbf{5}, \mathbf{3}]$ is possible; it can also be derived from the theorem of Gerstenhaber and Rothaus on solutions of nonsingular sets of equations over residually finite groups [1]. We give here a direct proof, based on a construction used by Levin [2], for which only elementary knowledge of groups and monoids is required.

For an alphabet (set of symbols) $\Sigma, \Sigma^{*}$ denotes the free monoid with generators $\Sigma$, with the identity denoted by $e$.

A Thue system with a single rule is a set $T=\{(u, v)\}$ consisting of a pair of words over an alphabet $\Sigma$. The congruence $\stackrel{*}{\leftrightarrow}$ on $\Sigma^{*}$ associated with such a system $T$ is defined as follows: for any strings $x, y \in \Sigma^{*}$, define $x u y \leftrightarrow x v y$, and define $\leftrightarrow$ to be the reflexive, symmetric and transitive closure of $\leftrightarrow$. The quotient $\Sigma^{*} / \stackrel{\leftrightarrow}{\leftrightarrow}$ of $\Sigma^{*}$ by the congruence is a monoid, the monoid presented by $\langle\Sigma \mid u=v\rangle$.

THEOREM. Let $\Sigma$ be an alphabet, $\Gamma$ a subset of $\Sigma$, and $u, v$ strings in $\Sigma^{*}$. Consider the Thue system $\{(u, v)\}$ with associated congruence $\stackrel{\leftrightarrow}{\leftrightarrow}$ and monoid $M=\Sigma^{*} / \stackrel{\leftrightarrow}{\leftrightarrow}$. If a letter not in $\Gamma$ occurs in $u$ or $v$ then for any $x, y \in \Gamma^{*}, x \leftrightarrow 4$ implies $x=y$. In other words, if uv $\notin \Gamma^{*}$ then the submonoid of $M$ generated by the congruence classes of $\Gamma$ is freely generated by them.

Proof. If both $u$ and $v$ contain letters not in $\Gamma$, then the rule $u \leftrightarrow v$ does not apply to any word in $\Gamma^{*}$ and the conclusion of the theorem is clearly true. Suppose, therefore, that $u$, but not $v$, contains letters not in $\Gamma$.

We may assume that $\Gamma$ includes all the letters of $\Sigma$ except one, say $a$. Suppose $u$ has some $n>0$ occurrences of $a$ and write $u$ as $w a u_{0} \cdots a u_{n-1}$, where $w$ and each $u_{i}$ are in $\Gamma^{*}$.

To establish the theorem, it is sufficient to prove that there is a group $G$ and a homomorphism $\phi: M \rightarrow G$ that is one-to-one on (the congruence classes of) $\Gamma$ such that $\phi(\Gamma)$ freely generates a free subgroup of $G$. Let $F$ be the free group on $\Gamma$, with

Received by the editors October 4, 1982 and, in revised form, February 3, 1983.

1980 Mathematics Subject Classification. Primary $20 \mathrm{M} 05$.

${ }^{1}$ This research was supported in part by the National Science Foundation under Grant No. MCS81-16327. A preliminary version was presented at the First International Conference for Combinatorics on Words, held at the University of Waterloo, August 1982.

(C) 1983 American Mathematical Society $0002-9939 / 83 \$ 1.00+\$ .25$ per page 
identity $1_{F}$. View the rule $u \leftrightarrow v$ as an equation " $a u_{0} \cdots u_{n-2} a\left(u_{n-1} v^{-1} w\right)=1$ " to be solved for the variable $a$ over $F$ : applying Levin's theorem [2], there is a group $G$ containing $F$ as a subgroup and an element $\bar{a}$ of $G$ such that $\bar{a} u_{0} \cdots \bar{a} u_{n-1} v^{-1} w=$ $1_{G}$. The homomorphism $\phi$ can then be defined via the inclusion of $\Gamma^{*}$ in $F$ with $\phi(a)=\bar{a}$, thus completing the proof.

For the Thue system $\{(u, v)\}$, Levin's construction takes the following form. The group $G$ is the wreath product of $F$ with the cyclic group $Z_{n}=\{0,1, \ldots, n-1\}$ : that is, elements of $G$ are pairs $(k, C)$ with $k \in Z_{n}$ and $C: Z_{n} \rightarrow F$ an arbitrary function; and multiplication is given by $\left(m, C_{1}\right) \cdot\left(k, C_{2}\right)=\left(m+k, C_{3}\right)$ where $C_{3}(i)=$ $C_{1}(i-k) C_{2}(i), 0 \leq i \leq n-1$, and the index computation is modulo $n$.

For $x \in \Gamma^{*}$ define $\hat{x}: Z_{n} \rightarrow F$ by $\hat{x}(i)=x, 0 \leq i \leq n-1$. Let $A(i)=u_{i}^{-1}$ for $0 \leq i \leq$ $n-2$ and let $A(n-1)=w^{-1} v u_{n-1}^{-1}$. Finally, let $h: \Sigma^{*} \rightarrow G$ be the homomorphism determined by defining $h(a)=(-1, A)$ and, for $b \in \Gamma, h(b)=(0, \hat{b})$. Note that for $x \in$ $\Gamma^{*}, h(x)=(0, \hat{x})$. (In the case $n=1$, this reduces to the homomorphism $h: \Sigma^{*} \rightarrow F$ given by $h(a)=w^{-1} v u_{0}^{-1}, h(b)=b$.)

It follows from the definition of $h$ that $h(u)=h(v)$, and therefore for any $x, y \in$ $\Sigma^{*}$, if $x \dot{\leftrightarrow} y$ then $h(x)=h(y)$. In particular, if $x, y \in \Gamma^{*}$ and $x \dot{\leftrightarrow} y$ then from $h(x)=$ $h(y)$ we conclude that $(0, \hat{x})=(0, \hat{y})$ and so $x=y$, as desired.

\section{REFERENCES}

1. M. Gerstenhaber and O. S. Rothaus, The solution of sets of equations in groups, Proc. Nat. Acad. Sci. U.S.A. 48 (1962), 745-750.

2. F. Levin, Solutions of equations over groups, Bull. Amer. Math. Soc. 68 (1962), 603-604.

3. R. C. Lyndon and P. E. Schupp, Combinatorial group theory, Springer-Verlag, Berlin and New York, 1977.

4. W. Magnus, Über diskontinuierliche Gruppen mit einer definierenden Relation (Der Freiheitssatz), J. Reine Angew. Math. 163 (1930), 141-165.

5. W. Magnus, A. Karass and D. Solitar, Combinatorial group theory, Wiley-Interscience, New York, 1966.

Department of Mathematics, University of California at Santa Barbara, Santa Barbara, California 93106 